\title{
Unlicensed NK cells target neuroblastoma following anti-GD2 antibody treatment
}

\author{
Nidale Tarek, ${ }^{1}$ Jean-Benoit Le Luduec,, ${ }^{2}$ Meighan M. Gallagher, ${ }^{2}$ Junting Zheng, ${ }^{3}$ \\ Jeffrey M. Venstrom, ${ }^{4}$ Elizabeth Chamberlain, ${ }^{1}$ Shakeel Modak, ${ }^{1}$ Glenn Heller, ${ }^{3}$ \\ Bo Dupont, ${ }^{2}$ Nai-Kong V. Cheung, ${ }^{1}$ and Katharine C. Hsu ${ }^{4,5}$
}

\begin{abstract}
${ }^{1}$ Department of Pediatrics, ${ }^{2}$ Immunology Program, Sloan-Kettering Institute, ${ }^{3}$ Department of Epidemiology-Biostatistics, and ${ }^{4}$ Department of Medicine, Memorial Sloan-Kettering Cancer Center, New York, New York, USA. ${ }^{5}$ Department of Medicine, Weill Cornell Medical College, New York, New York, USA.
\end{abstract}

\begin{abstract}
Survival outcomes for patients with high-risk neuroblastoma (NB) have significantly improved with anti-disialoganglioside GD2 $\mathrm{mAb}$ therapy, which promotes NK cell activation through antibody-dependent cell-mediated cytotoxicity. NK cell activation requires an interaction between inhibitory killer cell immunoglobulin-like receptors (KIRs) and HLA class I ligands. NK cells lacking KIRs that are specific for self HLA are therefore "unlicensed" and hyporesponsive. mAb-treated NB patients lacking HLA class I ligands for their inhibitory KIRs have significantly higher survival rates, suggesting that NK cells expressing KIRs for non-self HLA are mediating tumor control in these individuals. We found that, in the presence of $\mathrm{mAb}$, both licensed and unlicensed NK cells are highly activated in vitro. However, HLA class I expression on NB cell lines selectively inhibited licensed NK cell activity, permitting primarily unlicensed NK cells to mediate antibody-dependent cell-mediated cytotoxicity. These results indicate that unlicensed NK cells play a key antitumor role in patients undergoing $\mathrm{mAb}$ therapy via antibody-dependent cell-mediated cytotoxicity, thus explaining the potent "missing KIR ligand" benefit in patients with NB.
\end{abstract}

\section{Introduction}

Neuroblastoma (NB), an embryonal malignancy of neuroectodermal origin, is the most common extracranial solid tumor of childhood. Nearly two-thirds of patients present at diagnosis with evidence of metastatic disease and have poor long-term survival due to residual disease, despite aggressive approaches, including highdose multiagent chemotherapy, surgery, radiation therapy, and autologous stem cell transplantation (ASCT) $(1,2)$. Treatment of patients with high-risk NB with monoclonal antibodies $(\mathrm{mAb})$ targeting the disialoganglioside surface antigen GD2 has resulted in lower recurrence rates and improved overall survival (OS) (3-5). In addition to complement-dependent cytotoxicity, the anti-GD2 mAb 3F8 achieves NB killing through antibody-dependent cell-mediated cytotoxicity (ADCC) mediated by myeloid and NK cells (4).

NK activity is regulated by inhibitory and activating signals following engagement of cell membrane receptors with their cognate ligands on target cells (6). Different mechanisms of NK activation and inhibition have been described upon NK interaction with NB. Untreated NB tumors and cell lines are widely reported to have reduced to no HLA class I expression, rendering them potentially susceptible to NK killing due to lack of engagement of HLA class I-specific inhibitory killer cell immunoglobulin-like receptors (KIRs) $(7,8)$. In addition to CD16-mediated activation by $\mathrm{mAb}$, direct activation of NK cells by NB through NKp30, NKp44, NKp46, and the DNAM-1 receptor has also been described (9-11). To evade NK surveillance, $\mathrm{NB}$ cells exhibit poor cell surface expression of the activating ligands MICA, MICB, and ULBPs; and high serum concentrations of soluble MICA in patients with NB results in depressed

Authorship note: Nidale Tarek and Jean-Benoit Le Luduec contributed equally to this work.

Conflict of interest: The authors have declared that no conflict of interest exists. Citation for this article: J Clin Invest. 2012;122(9):3260-3270. doi:10.1172/JCI62749.
NK function (8). Furthermore, the 4Ig-B7-H3 molecule, widely expressed among solid tumors, including NB $(12,13)$, is a potent inhibitor of NK function (14).

NK cells spare autologous cells from killing through interaction of inhibitory receptors with self-HLA class I antigens on the autologous cell. KIR2DL2 and KIR2DL3 recognize HLA-C allotypes characterized by $\mathrm{Asn}^{80}$ (grouped as HLA-C1); KIR2DL1, and to a weaker extent some KIR2DL2/3 allotypes, recognize HLA-C allotypes characterized by Lys $^{80}$ (grouped as HLA-C2); KIR3DL1 recognizes HLA-A and HLA-B allotypes with the Bw4 epitope; and the heterodimeric CD94/NKG2A receptor recognizes complexes of HLA-E bound to peptides from the leader sequences of HLA class I molecules $(15,16)$. Interaction between self-specific inhibitory KIRs and their cognate HLA ligands is fundamental to a process referred to as licensing (17), in which NK cells expressing inhibitory KIRs for self HLA (S-KIRs) are "licensed" and have higher resting capacity for response (IFN- $\gamma$ production, cytotoxicity, and ADCC) $(18,19)$. Lacking S-KIRs, "unlicensed" NK cells are significantly less responsive at rest and include cells expressing inhibitory KIRs for non-self HLA (NS-KIR). Under inflammatory conditions, however, unlicensed NK cells can exhibit higher response (20-22). Independent of KIRs, CD94/NKG2A expression is associated with modest response capacity (19). Because the KIR and HLA genes are located on different chromosomes and segregate independently, approximately $60 \%$ of individuals have inhibitory KIRs for which they lack the cognate HLA class I ligands (a condition referred to herein as missing KIR ligand) $(23,24)$ and therefore potentially possess substantial numbers of unlicensed NK cells expressing NS-KIRs.

We previously reported a significant association between KIR/ HLA genotypes predictive of missing KIR ligand and survival in 169 patients with NB treated with the anti-GD2 mAb 3F8 following ASCT (25). A smaller study of 38 patients treated with Hu14.18-IL2, an anti-GD2 mAb fused to IL-2, also suggested improved response among patients missing KIR ligand (26). The improved clinical outcomes suggest that unlicensed NK cells expressing NS-KIRs may play 
Table 1

Patient characteristics

\begin{tabular}{|c|c|c|}
\hline Characteristics & $\begin{array}{l}\text { Patients treated with chemotherapy } \\
\text { plus 3F8 }(n=76)\end{array}$ & $\begin{array}{l}\text { Patients treated with ASCT } \\
\text { plus 3F8 }(n=166)\end{array}$ \\
\hline Stage IV cancer & 75 (99\%) & $166(100 \%)$ \\
\hline $\begin{array}{l}\text { Age } \\
<1.5 \text { years } \\
>1.5 \text { years }\end{array}$ & $\begin{array}{c}6(8 \%) \\
70(92 \%)\end{array}$ & $\begin{array}{c}15(9 \%) \\
151(91 \%)\end{array}$ \\
\hline $\begin{array}{l}\text { MYCN status } \\
\text { Nonamplified } \\
\text { Amplified } \\
\text { Unknown }\end{array}$ & $\begin{array}{l}45(59 \%) \\
21(28 \%) \\
10(13 \%)\end{array}$ & $\begin{array}{l}95(57 \%) \\
55(33 \%) \\
16(10 \%)\end{array}$ \\
\hline $\begin{array}{l}\text { Lactate dehydrogenase } \\
<1,500 \text { units } / \mathrm{mol} \\
\geq 1,500 \text { units } / \mathrm{mol} \\
\text { Unknown }\end{array}$ & $\begin{array}{l}43(57 \%) \\
20(26 \%) \\
13(17 \%)\end{array}$ & $\begin{array}{l}83(50 \%) \\
45(27 \%) \\
38(23 \%)\end{array}$ \\
\hline $\begin{array}{l}\text { Bone metastases } \\
\text { No } \\
\text { Yes }\end{array}$ & $\begin{array}{l}17(22 \%) \\
59(78 \%)\end{array}$ & $\begin{array}{c}37(22 \%) \\
129(78 \%)\end{array}$ \\
\hline $\begin{array}{l}\text { Disease status at } 3 F 8 \text { th } \\
\text { First CR/VGPR } \\
\text { Second CR/VGPR } \\
\text { Primary refractory } \\
\text { Secondary refractory } \\
\text { Others }\end{array}$ & $\begin{array}{c}26(34 \%) \\
2(3 \%) \\
47(62 \%) \\
0(0 \%) \\
1(1 \%)\end{array}$ & $\begin{array}{c}80(48 \%) \\
17(10 \%) \\
41(25 \%) \\
3(2 \%) \\
25(15 \%)\end{array}$ \\
\hline $\begin{array}{l}\text { KIR genotype } \\
\text { 3DL1 present } \\
\text { 2DL2 present } \\
\text { 2DL3 present } \\
\text { 2DL1 present }\end{array}$ & $\begin{array}{l}73(96 \%) \\
44(58 \%) \\
65(86 \%) \\
75(99 \%)\end{array}$ & $\begin{array}{c}157(95 \%) \\
80(48 \%) \\
151(91 \%) \\
163(98 \%)\end{array}$ \\
\hline $\begin{array}{l}\text { KIR/HLA ligand } \\
\text { All ligands present } \\
\text { Missing } \geq 1 \text { ligand }\end{array}$ & $\begin{array}{l}29(38 \%) \\
47(62 \%)\end{array}$ & $\begin{array}{c}60(36 \%) \\
106(64 \%)\end{array}$ \\
\hline
\end{tabular}
VGPR, complete response/very good partial response.
APatients who received $3 \mathrm{~F} 8$ as part of induction therapy in combination with chemotherapy. CR/

sary for this benefit, we evaluated updated outcomes of 166 previously reported patients who received $3 \mathrm{~F} 8$ following ASCT (25) and 76 new patients who received 3F8 following chemotherapy without ASCT. Patient characteristics for both cohorts are listed in Table 1 . The median followup was 74 months from the institution of 3F8 immunotherapy, and the OS and PFS were similar in both patient groups (data not shown).

Patients were considered missing KIR ligand if they lacked one or more HLA class I ligand for their inhibitory KIRs. In contrast, patients with "all ligands present” possessed all HLA class I ligands for their inhibitory KIRs. 153 patients (63\%) were missing KIR ligand, and the proportions of missing KIR ligand were comparable among patients who received chemotherapy without ASCT (62\%) and those who received ASCT (64\%). In both the non-ASCT and ASCT settings, missing KIR ligand was associated with increased OS and PFS (Figure 1, A and B), indicating that ASCT is not necessary for the "missing ligand" effect and suggesting that the effect may be more related to the $\mathrm{mAb}$ $3 \mathrm{~F} 8$ or to NK-NB interaction in general. Among patients receiving 3F8, the median OS and PFS were 114 and 50 months, respectively, for patients missing KIR ligand compared with a median OS and PFS of 51 and 18 months, respectively, for patients with all ligands present (Figure 1C). Thus, compared with that of patients with all KIR ligands, patients lacking one or more class I ligands for autologous inhibitory KIRs had significantly longer

an important role in patients treated with $\mathrm{mAb}$. In this study, we confirm the impact of missing KIR ligand in patients with NB receiving 3F8 and demonstrate that ASCT is not necessary for this association. We demonstrate that $3 \mathrm{~F} 8$ in vitro activates NK cells expressing S-KIRs, NS-KIRs, and NKG2A for ADCC, but cytokine-induced upregulation of HLA class I expression on NB targets selectively abrogates activation of S-KIR-positive and NKG2A-positive NK cells. These results not only demonstrate the dominance of the unlicensed NS-KIR-positive NK cell in ADCC and antitumor cytotoxicity but also confirm that missing KIR ligand KIR/HLA genotype combinations represent an important prognostic marker in patients with NB treated with mAb. KIR/HLA genotypes indicative of missing KIR ligand may therefore be a positive prognostic indicator for other patient populations with malignancies treated with $\mathrm{mAb}$.

\section{Results}

Missing KIR ligand is associated with improved survival in patients with bigh-risk $N B$ receiving anti-GD2 $m A b$. We previously reported that patients receiving ASCT with 3F8 had superior OS and progression-free survival (PFS) if they lacked HLA class I ligands for autologous inhibitory KIRs (25). To test whether ASCT is neces-
OSs (hazard ratio [HR] $=0.57$ [95\% CI, 0.39-0.83], $P=0.003$ ) and PFSs $(\mathrm{HR}=0.58$ [95\% CI, 0.42-0.81], $P=0.001)$. In the multivariate analysis, which controlled for age, MYCN amplification, and bone metastases, the missing ligand effect was maintained for OS $(\mathrm{HR}=0.48$ [95\% CI, 0.32-0.71], $P<0.001)$ and PFS $(\mathrm{HR}=0.52$ (95\% CI, 0.36-0.74), $P<0.001$ ) (Table 2 ), and there were no significant differences in disease status between the missing KIR ligand and all ligands present groups $(P=0.66$; Supplemental Table 1 ; supplemental material available online with this article; doi:10.1172/ JCI62749DS1). Although lack of any one class I ligand was associated with improved survival over that of those who possessed all KIR ligands, the highest OSs ( $\mathrm{HR}=0.38$ [95\% CI, 0.18-0.79], $P=0.01$ ) and PFSs $(\mathrm{HR}=0.28$ [95\% CI, 0.13-0.58], $P=0.001)$ were evident in patients who were HLA-Bw6/Bw6 and lacking the HLA-Bw4 ligand for autologous KIR3DL1 (Supplemental Figure 1).

Licensed and unlicensed NK cells are activated in the presence of $m A b$ 3F8. The improved outcome in patients with high-risk NB missing KIR ligand implies that unlicensed NK cells expressing NS-KIRs in these individuals become activated and contribute significantly to tumor control. To determine whether unlicensed NK cells expressing NS-KIRs can be mobilized by 3F8 for ADCC and tumor toxic- 
A

No ASCT

B

C

ASCT
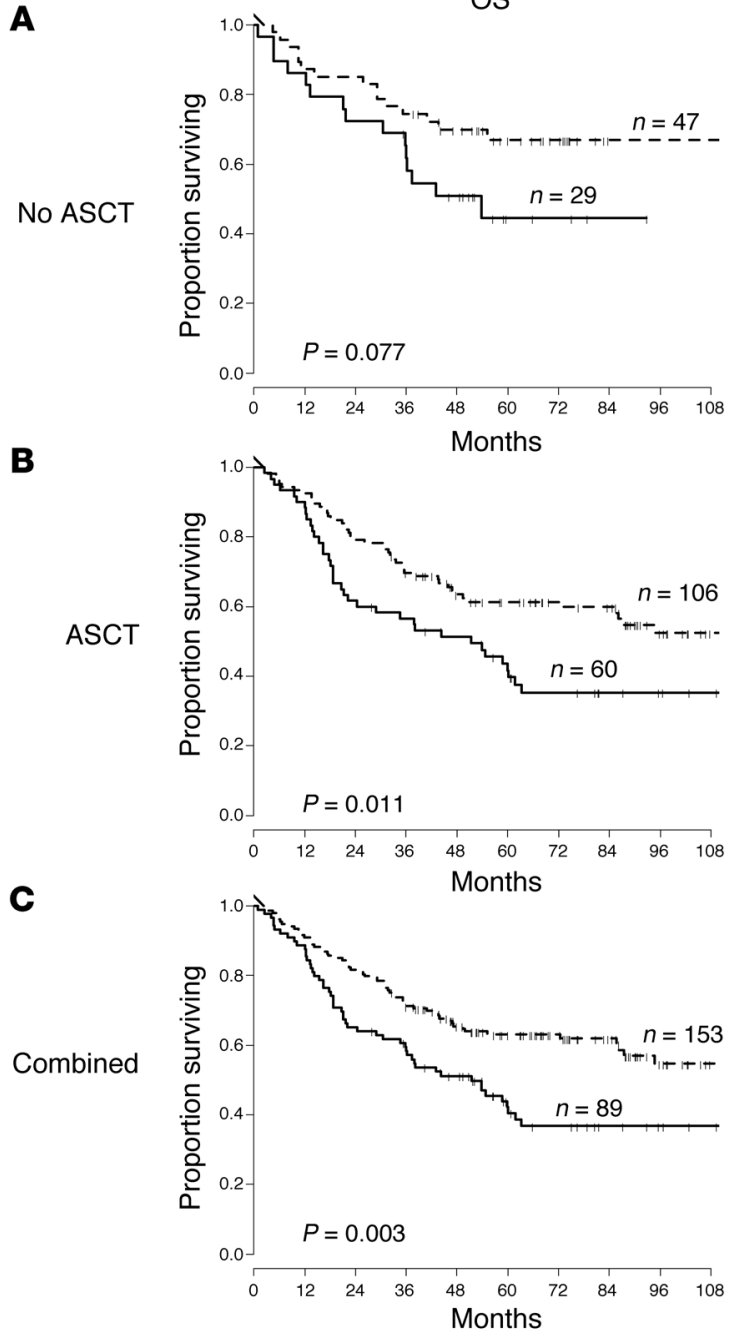

PFS
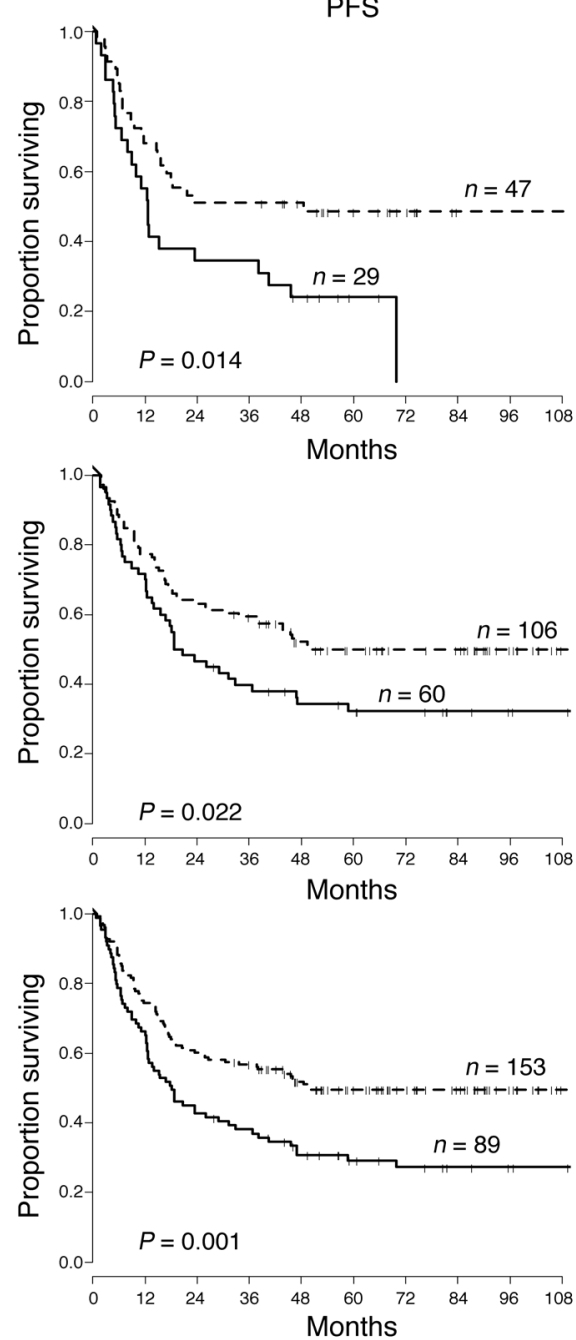

\section{Figure 1}

Missing KIR ligand is associated with improved OS and PFS in patients with high-risk NB treated with 3F8. Among patients receiving 3F8, patients lacking class I ligands for autologous inhibitory KIRs (dotted lines) have higher OS and PFS compared with those of patients with all KIR ligands present (solid lines) following (A) chemotherapy alone (OS, HR $=0.53[95 \% \mathrm{Cl}, 0.26-1.08], P=0.077 ; \mathrm{PFS}, \mathrm{HR}=0.49[95 \% \mathrm{Cl} 0.28-0.88]$, $P=0.014$ ) or (B) ASCT (OS, HR $=0.57$ [95\% Cl, 0.37-0.88], $P=0.011$; PFS, $\mathrm{HR}=0.62$ [95\% Cl, 0.41-0.94], $P=0.022$ ), confirming that ASCT is not necessary for the missing ligand effect. (C) Among all patients receiving 3F8, patients missing KIR ligands have higher OS (HR $=0.57[95 \%$ $\mathrm{Cl}, 0.39-0.83], P=0.003)$ and $\mathrm{PFS}(\mathrm{HR}=0.58[95 \% \mathrm{Cl}, 0.42-0.81], P=0.001)$ compared with those of patients with all $\mathrm{KIR}$ ligands.

ity in vitro, we evaluated unlicensed and licensed NK cell activity from normal individuals in response to NB cell lines in the presence or absence of 3F8. Donors with KIR and HLA genotypes predictive of missing KIR ligand $(n=16)$ were selected for evaluation, allowing functional comparisons between NK populations expressing S-KIRs or NS-KIRs within each individual. Because donors were of a variety of KIR and HLA ligand backgrounds, licensed and unlicensed NK populations were defined by different KIR/self-HLA combinations (Supplemental Table 2). Four donors with all ligands present were also evaluated.

Multicolor flow cytometry provided single-cell assessment of NK cells exclusively expressing a single inhibitory KIR, permitting evaluation of unlicensed NK cells expressing NS-KIRs distinct from licensed NK cells expressing S-KIRs. Among individuals with missing KIR ligand, unlicensed NK cells exclusively express- ing a single NS-KIR represented 3\%-35\% of the total NK repertoire (Supplemental Table 3). Among these same individuals, licensed NK cells exclusively expressing a single S-KIR represented $8 \%-31 \%$ of the total NK repertoire. In individuals with all ligands present, licensed NK cells exclusively expressing 1 S-KIR represented $19 \%-$ $35 \%$ of the total NK repertoire. Thus, unlicensed and licensed NK cells expressing a single inhibitory KIR can represent substantial segments of the total NK repertoire.

In each individual, we compared CD107 mobilization as a marker of cytotoxic response among NS-KIR-positive and S-KIR-positive NK cells following stimulation with the HLA class I-negative K562 target cell line or with HLA-genotyped NB cell lines (Supplemental Table 4) in the presence or absence of mAb 3F8. Among all individuals, both NS-KIR and S-KIR NK cell populations had dim CD56 expression, indicating their equiva- 


\begin{tabular}{lcccccccc} 
& \multicolumn{2}{c}{ Univariate analysis of OS } & \multicolumn{2}{c}{ Multivariate analysis of OS } & \multicolumn{2}{c}{ Univariate analysis of PFS } & \multicolumn{2}{c}{ Multivariate analysis of PFS } \\
Variable & HR (95\% Cl) & $\boldsymbol{P}$ value & HR (95\% CI) & $\boldsymbol{P}$ value & HR (95\% CI) & $\boldsymbol{P}$ value & HR (95\% CI) & $\boldsymbol{P}$ value \\
Missing any ligand & $0.57(0.39-0.83)$ & 0.003 & $0.48(0.32-0.71)$ & $<0.001$ & $0.58(0.42-0.81)$ & 0.002 & $0.52(0.36-0.74)$ & $<0.001$ \\
Age $\geq 1.5$ years & $2.53(1.03-6.22)$ & 0.043 & $2.32(0.92-5.85)$ & 0.074 & $1.89(0.92-3.86)$ & 0.081 & $1.58(0.75-3.29)$ & 0.226 \\
Bone disease & $1.20(0.75-1.91)$ & 0.449 & $1.10(0.67-1.81)$ & 0.709 & $1.48(0.96-2.28)$ & 0.078 & $1.38(0.87-2.18)$ & 0.175 \\
MYCN amplification & $1.04(0.69-1.56)$ & 0.868 & $1.19(0.78-1.82)$ & 0.407 & $1.06(0.73-1.53)$ & 0.758 & $1.18(0.81-1.71)$ & 0.397 \\
\hline
\end{tabular}

lent terminal differentiation and potential for cytotoxic response $(27,28)$. Furthermore, there was no difference in CD16 expression among the NK populations (data not shown).

As expected, in an HLA-C1/C1, HLA-Bw4/Bw6 individual, licensed NK cells exclusively expressing the self-specific KIR2DL3 or KIR3DL1 receptor were responsive to K562 cells, while unlicensed NK cells expressing the non-self-specific KIR2DL1 receptor were hyporesponsive (Figure 2A). Although NB cell lines have low to no cell surface expression of HLA class I molecules $(7,8)$ (Figure $3 \mathrm{~A}$ ), rendering them potentially susceptible to NK cytotoxicity, all NK cells, regardless of their licensed status, responded poorly to stimulation with the NB cell line LAN-1 (Figure 2A). In the presence of the anti-GD2 mAb 3F8, however, licensed single-positive NK (spNK) cells for S-KIRs were highly activated when stimulated (Figure 2A). Unexpectedly, the unlicensed NS-KIR spNK cells also demonstrated higher activation in the presence of 3F8, compared with that of the K562 control. KIR-negative NK cells were also activated but to a lesser degree. These results were confirmed with the IMR-32 cell line and 4 other NB cell lines (data not shown), demonstrating that multiple NK subsets are recruited by $3 \mathrm{~F} 8$ to mediate ADCC against NB target cells. Cytotoxicity assays confirmed that addition of 3F8 was critical for NK-mediated NB lysis (Supplemental Figure 2).

Aggregate analysis of NK response to the NB cell line, LAN-1, from 16 individuals demonstrated that NK cells expressing S-KIRs or NS-KIRs showed strong CD107 mobilization mediated by $3 \mathrm{~F} 8$ $(P<0.0001)$ (Figure 2B). A higher percentage of S-KIR spNK cells compared with NS-KIR spNK cells exhibited CD107 mobilization $(P=0.004)$, confirming that licensed NK cells are more activated for $\operatorname{ADCC}(18,19)$. The aggregate analysis also showed response among KIR-negative NK cells, albeit to a lower level than that in the NS-KIR-positive population $(P=0.006)$.

Based on the results in the KIR-negative NK cells, we surmised that CD94/NKG2A may contribute to the NK cell response to LAN-1 cells in the presence of $3 F 8$. Indeed, NKG2A expression contributed to response among all NK cell populations, including the KIR-negative cells (Figure 2C). Among individuals with missing KIR ligand, NKG2A-positive KIR-negative NK cells represented $13 \%-51 \%$ of the total NK repertoire, and there was no preferential coexpression of NKG2A among NS-KIR or S-KIR populations (data not shown).

HLA class I expression on tumor cells selectively inbibits licensed NK cells, sparing ADCC by unlicensed NK cells. In vitro, NK cells exclusively expressing S-KIRs or NS-KIRs are activated by 3F8 for ADCC against $\mathrm{NB}$, although comparatively S-KIR-positive NK cells have higher response. In vivo, however, higher survival among patients missing KIR ligands for autologous inhibitory KIRs suggests not only that unlicensed NK cells expressing NS-KIRs are major contributors to tumor control but also that licensed NK cells expressing S-KIRs in patients with all ligands present are inhibited or silenced. Others have shown that HLA class I molecules, including the nonclassical HLA-E, are upregulated on NB cells following exposure to chemotherapy, isotretinoin, and anti-GD2 antibodies (29-32), therapeutic agents that the patients with high-risk NB in this study received. We therefore hypothesized that upregulation of self-HLA class I ligands on NB cells specifically inhibits licensed NK cells expressing inhibitory receptors for self-HLA ligands while sparing unlicensed NK cells lacking self-specific inhibitory receptors.

To isolate the role of KIR and HLA in NK-NB interactions, we evaluated NKG2A-negative NK cells for CD107 mobilization and IFN- $\gamma$ production in response to NB cell lines and $3 \mathrm{~F} 8$ with induced self-HLA expression. HLA class I expression on NB cells was induced following culture for 72 hours with IFN- $\gamma$ (33). Reproducing autologous NK-NB interactions in a patient missing KIR ligand, NK cells from an HLA-C1/C1/Bw6/Bw6 individual were incubated with the target LAN-1 cells. Although the HLA genotype of LAN-1 cells is HLA-C1/C1/Bw4/Bw6, IFN- $\gamma$-induced HLA class I expression did not include HLA-Bw4 (Figure 3A), therefore mimicking an HLAC1/C1/Bw6/Bw6 tumor target. While both S-KIR-positive and NS-KIR-positive NK cells were activated by LAN-1 cells with 3F8, upregulation of HLA expression on the target cell led to selective inhibition of the self-KIR2DL3 spNK cells (Figure 3B). In contrast, the non-self-specific KIR2DL1 and KIR3DL1 spNK cells maintained their level of activation in the presence of the class I-expressing tumor target. Reproducing autologous NK-NB interactions in an individual with all ligands present, NK cells from an HLA-C1/ $\mathrm{C} 2 / \mathrm{Bw} 4 / \mathrm{Bw} 4$ individual were incubated with the HLA-C1/C2/Bw4/ $\mathrm{Bw} 4$ cell line $\mathrm{BE}(2) \mathrm{N}$. IFN- $\gamma$ induced HLA class I expression, including HLA-Bw4, on the BE(2)N target cell line (Figure 3A). Licensed NK cells exclusively expressing KIR2DL1, KIR2DL3, or KIR3DL1 were activated by $\mathrm{BE}(2) \mathrm{N}$ cells in the presence of $3 \mathrm{~F} 8$, and all were inhibited by induced HLA class I expression on the target cell (Figure $3 \mathrm{~B}$ ). Confirming that the inhibition was due to interaction of cognate HLA class I molecules on the target cells with self-specific KIRs on the licensed NK cells, addition of anti-HLA class I blocking antibodies to the effector/target mix fully restored activity among the S-KIR NK cells, while leaving activity among the NS-KIR NK cells unchanged (Figure 3B). Selective inhibition of licensed NK cells by induced cognate HLA class I ligands was verified with the NB-1691 and IMR-32 cell lines (data not shown).

In the aggregate analysis of unlicensed and licensed NK response to LAN-1 cells from 9 individuals homozygous for HLA-C1 and therefore lacking KIR ligand HLA-C2, HLA class I upregulation on the NB target cell led to selective inhibition of CD107 mobilization among self-specific KIR2DL2 $/ 3^{+}$cells and, consequently, a stronger relative response in the non-self-specific KIR2DL $1^{+}$cells $(P=0.003$, 
A

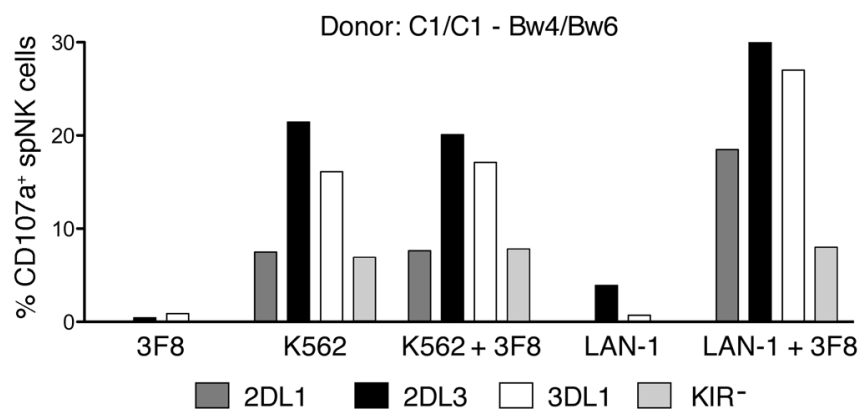

B

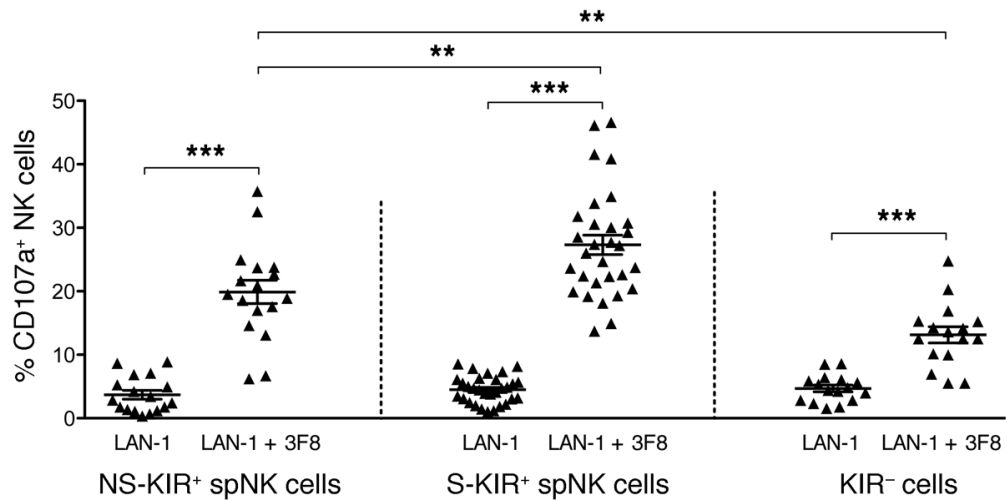

C

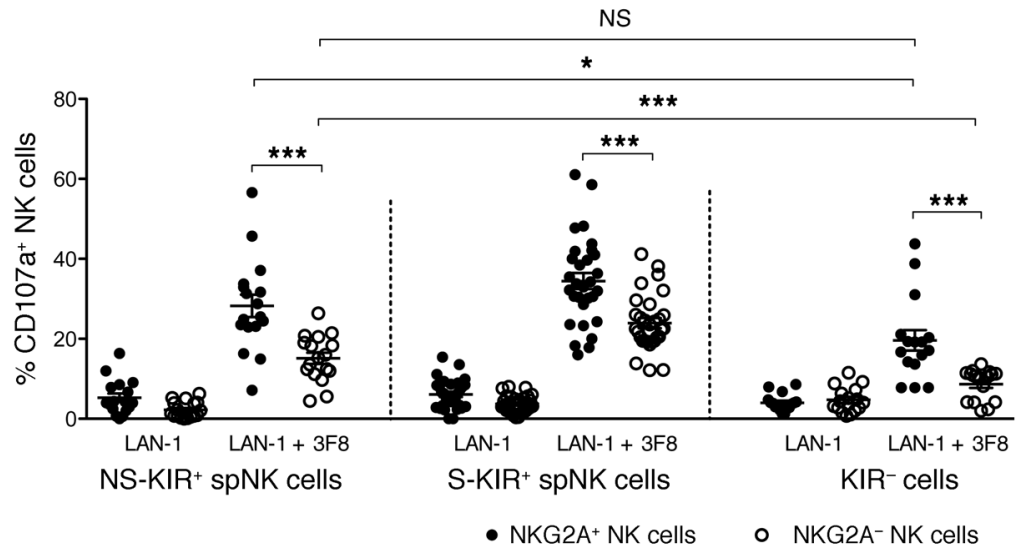

Figure 2

3F8 activates NK cells expressing S-KIRs, NSKIRs, and NKG2A against NB targets. In healthy individuals, CD107 degranulation was analyzed in the presence of NB target cells (LAN-1 cells), with and without $\mathrm{mAb}$, among subsets of NK cells. (A) In individual no. 8, S-KIR spNK cells demonstrate strong activation in response to K562 compared with that of NS-KIR spNK cells. All NK populations show minimal activation in response to LAN-1 cells alone but are activated by the addition of 3F8 in response to LAN-1 cells. Data represent the average of 3 separate experiments. (B) Aggregate function of S-KIR and NS-KIR spNK cells from 16 healthy individuals. Addition of 3F8 to LAN-1 cells results in activation of S-KIR-positive, NS-KIR-positive, and KIR-negative NK cells $(P<0.0001)$, with S-KIR spNK cells more responsive $(P=0.004)$ and KIR-negative NK cells less responsive $(P=0.006)$ than NS-KIR spNK cells. (C) NKG2A expression contributes to NK response to LAN-1 cells in the presence of $3 F 8$ among NK cells expressing S-KIR, and NS-KIR $(P<0.0001)$, and KIR-negative NK cells $(P=0.0004)$. NS-KIR-positive, NKG2A-negative NK cells are more responsive than KIR-negative, NKG2A-negative NK cells $(P=0.0008)$ and are equally responsive to KIRnegative, NKG2A-positive cells $(P=0.13)$. Symbols represent individual samples (mean \pm SEM). ${ }^{\star} P<0.05,{ }^{* *} P<0.01,{ }^{* \star} P<0.001$.
Figure 3C). Among NKG2A-negative cells, NS-KIR-positive NK cells were more responsive than KIR-negative NK cells $(P=0.001)$. Similar results were obtained with intracellular IFN- $\gamma$ response (data not shown). Aggregate analysis of NK activity among 3 individuals with all ligands present was consistent, with inhibition of all S-KIR-bearing NK subsets by NB target cells expressing the cognate HLA class I ligands ( $P<0.0001$, Figure $3 C)$.

When NKG2A-positive cells were evaluated, NKG2A coexpression enhanced ADCC response of all NK cell subsets, but it did not prevent HLA class I-mediated inhibition of licensed cells (Supplemental Figure 3). Moreover, NKG2A-positive cells were also inhibited by HLA-E expression on the $\mathrm{BE}(2) \mathrm{N}$ target cell, as evidenced by suppression of activity among the NKG2A-positive KIR-negative subset (Supplemental Figure 3).

Cytokines released by activated NK cells induce HLA class I expression on NB cell lines. Induced HLA expression has been described in NB tumors isolated from patients receiving treatment (29). To determine whether upregulation of HLA class I molecules on NB target cell lines is directly induced by agents commonly used in the treatment of NB, the NB cell lines LAN-1 and BE(2) $\mathrm{N}$ were incubated with GM-CSF, isotretinoin, or with different chemotherapy agents. No increase in HLA class I expression on the NB cell lines was detected following treatment with these agents (data not shown). We then investigated whether cytokines released from NK cells activated by 3F8-dependent ADCC could induce HLA class I expression on NB cells. PBMCs and NB targets were coincubated in the presence of $3 \mathrm{~F} 8$, with or without GM-CSF, which enhances 3F8-dependent ADCC (34). Supernatant obtained following 24 hours of coculture was collected and used as a culture medium for the NB cell lines LAN-1 and BE(2) $\mathrm{N}$ (Figure 4). Supernatant from PBMCs alone did not affect HLA class I expression on NB cell lines, and the addition of GM-CSF to PBMCs resulted in supernatant that minimally increased HLA class I expression on the NB target. Coincubation of 3F8 with PBMCs and NB cells, however, produced a supernatant capable of inducing HLA class I expression on LAN-1 and BE(2)N cells 
A
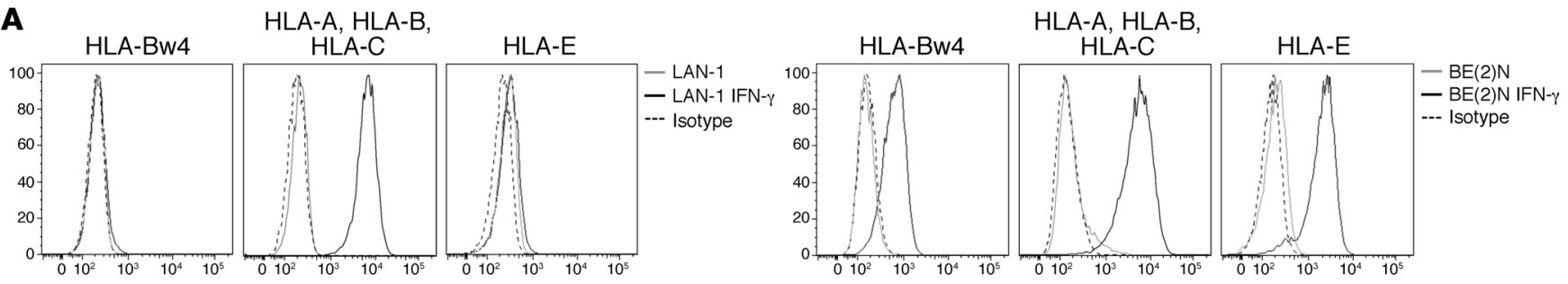

B
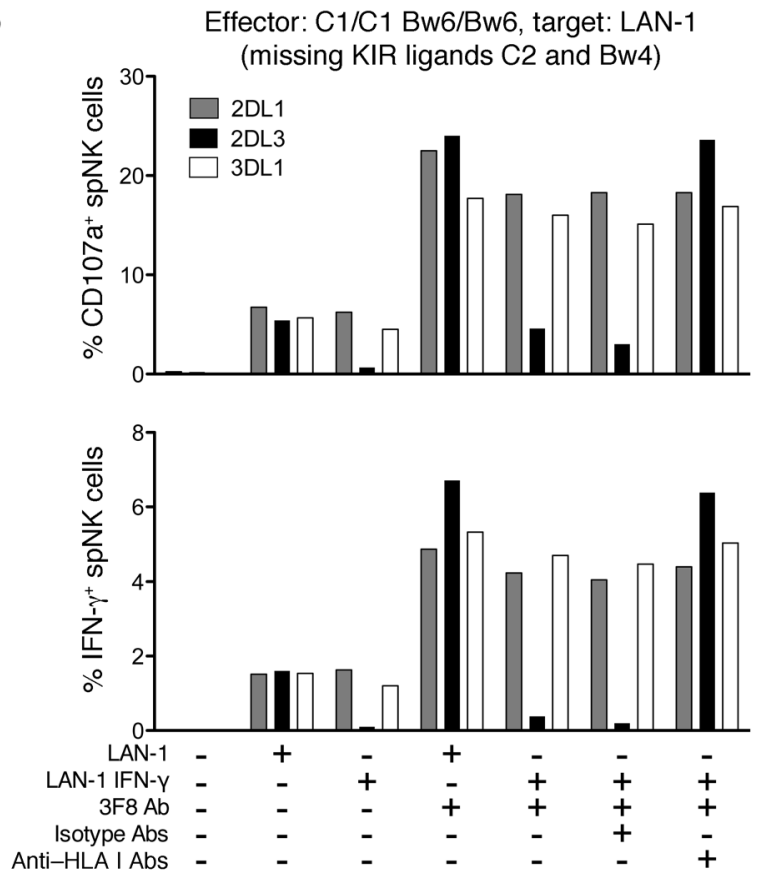

Effector: C1/C2 Bw4/Bw4, target: $\mathrm{BE}(2) \mathrm{N}$

(all ligands present)
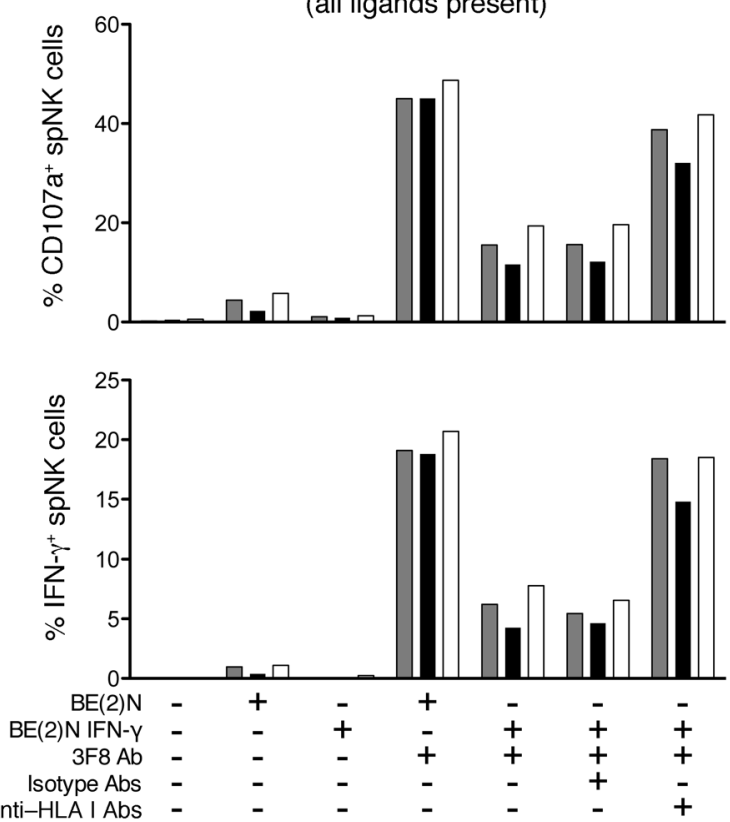

C

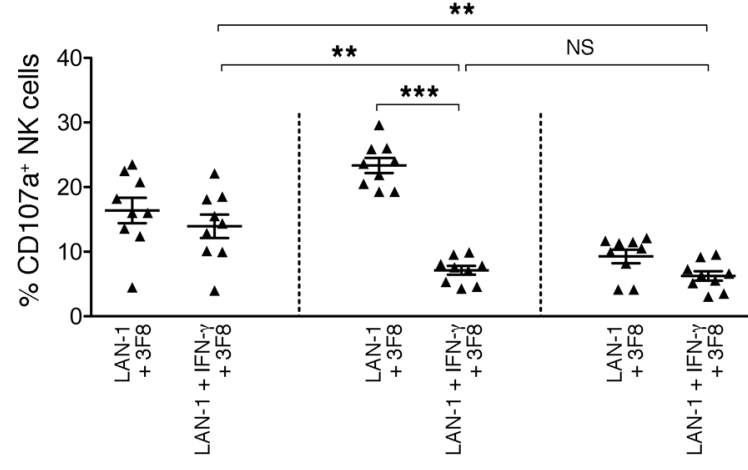

NS-KIR+ spNK cells S-KIR+ spNK cells

$\mathrm{KIR}^{-}$cells

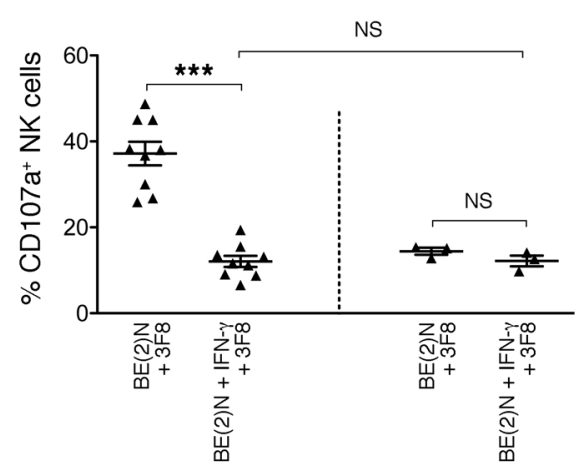

S-KIR+ spNK cells

$\mathrm{KIR}^{-}$cells

\section{Figure 3}

Licensed NK cells are selectively inhibited by HLA class I ligands induced on NB targets. (A) Incubation with IFN- $\gamma$ results in increased HLA class I antigen expression on LAN-1 and BE(2)N cells. HLA-Bw4 and HLA-E antigens are readily induced on BE(2)N but not LAN-1 cells. (B) CD107 degranulation and IFN- $\gamma$ production in NKG2A-negative, KIR-positive NK cells in response to IFN- $\gamma$-treated NB targets in the presence of $3 F 8$. When NK cells from individual no. 17 (HLA-C1/C1/Bw6/Bw6) are incubated with LAN-1 cells, mimicking effectors and NB cells in a patient lacking HLA-C2 and HLA-Bw4 ligands, both NS-KIR-positive (KIR2DL1 single positive [KIR2DL1sp] and KIR3DL1sp) and S-KIR-positive (KIR2DL3sp) NK cells are activated in the presence of 3F8, but only KIR2DL3 spNK cells are inhibited by IFN- $\gamma$-induced expression of self HLA on tumor target. When NK cells from individual no. 20 (HLA-C1/C2/Bw4/Bw4) are incubated with BE(2)N cells, mimicking effectors and NB cells in a patient with all ligands present, S-KIR-positive NK subsets (KIR2DL1sp, KIR2DL3sp, and KIR3DL1sp) are inhibited by IFN- $\gamma$-induced expression of cognate ligands on the tumor target. In both individuals, blocking antibodies to HLA class I fully restores response among S-KIR-positive NK cells. (C) Aggregate CD107 response among NK populations from 9 HLA-C1/C1 individuals to LAN-1 cells. HLA class I upregulation on the target inhibits S-KIR spNK cells, resulting in a comparatively stronger response from NS-KIR spNK cells $(P=0.003)$. Aggregate CD107 response among spNK cells from $3 \mathrm{HLA}-\mathrm{C} 1 / \mathrm{C} 2 / \mathrm{Bw} 4$ individuals to $\mathrm{BE}(2) \mathrm{N}$ demonstrates that HLA class I upregulation on the target inhibits all self-specific NK subsets. Symbols represent individual samples (mean \pm SEM). ${ }^{* *} P<0.01,{ }^{* \star *} P<0.001$. 
A

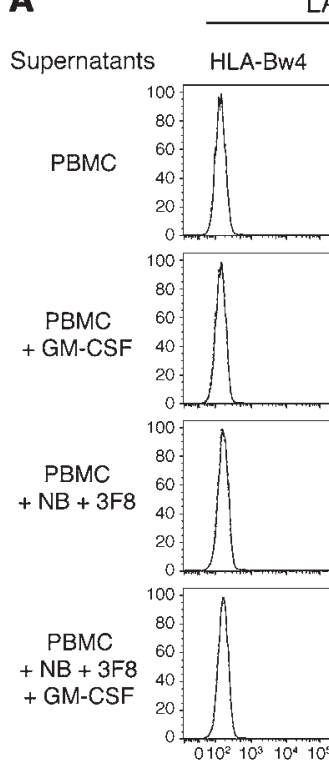

C

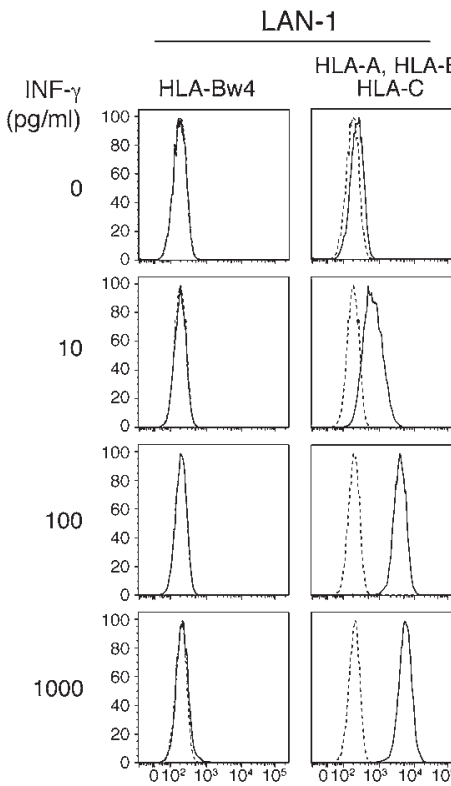

LAN-1

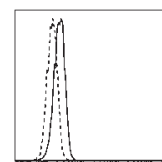

LAN-1 HLA-A, HLA-B
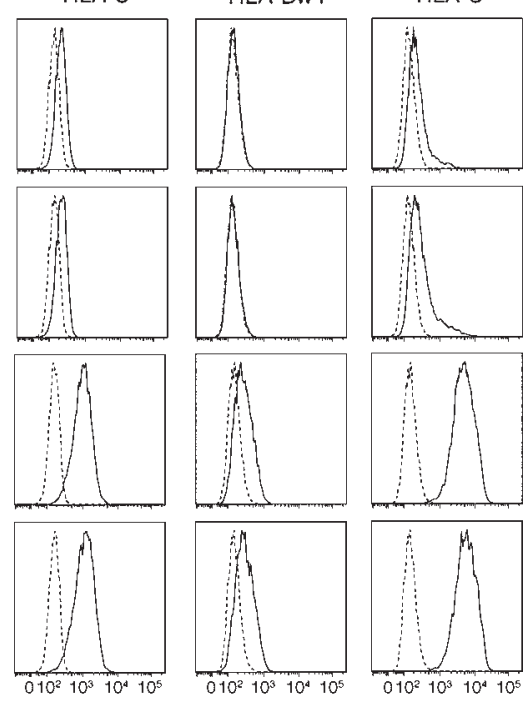
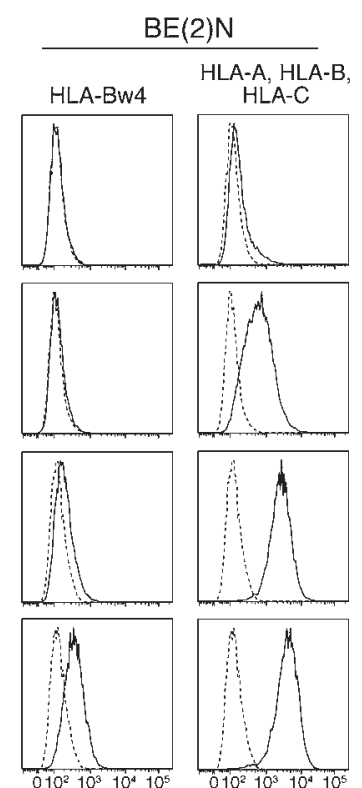

HLA-A, HLA-B,
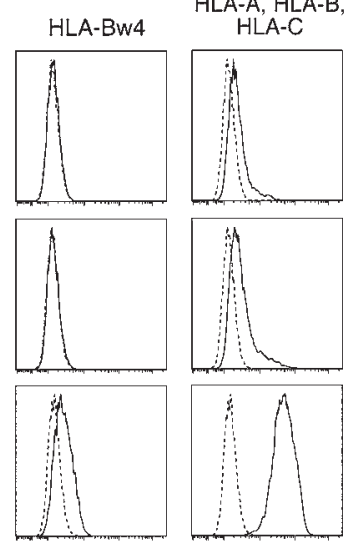

$\mathrm{BE}(2) \mathrm{N}$
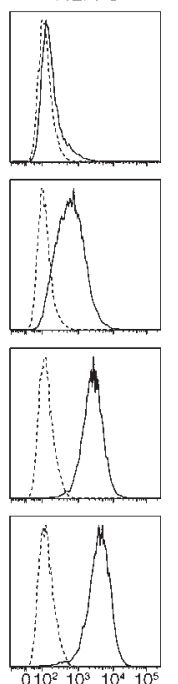
Isotype $\mathrm{Ab}$---

Anti-HLA I Ab-

B

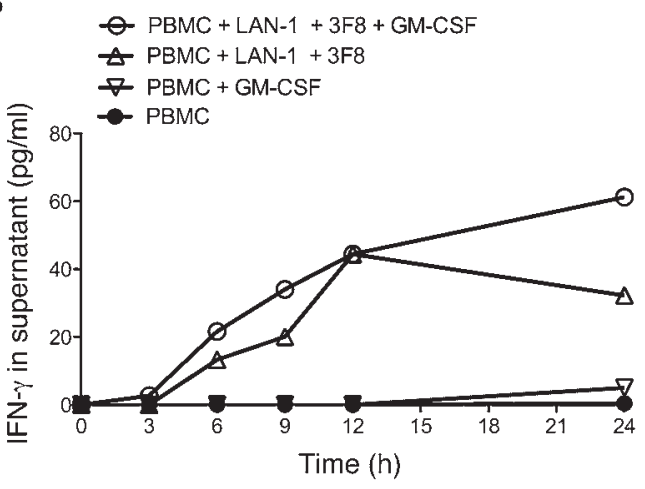

Anti-HLA I Ab Isotype $\mathrm{Ab}$-..

\section{Figure 4}

IFN- $\gamma$ released by activated NK cells induces upregulation of HLA class I on NB cells in vitro. (A) HLA-Bw4 and HLA-A, HLA-B, and HLA-C expression on the LAN-1 and $B E(2) N$ NB cell lines is shown following 72 hours in different culture conditions. Supernatants collected from PBMCs coincubated with LAN-1 and BE(2)N cells and 3F8, with or without GM-CSF, induced HLA class I expression on LAN-1 and $\mathrm{BE}(2) \mathrm{N}$ cells, respectively; in comparison, supernatant collected from PBMCs incubated with or without GM-CSF did not induce HLA expression. (B) By ELISA, PBMCs alone or PBMCs with GM-CSF produced no or minimal IFN- $\gamma$. PBMCs activated by LAN-1 cells in the presence of $3 F 8$ and GM-CSF released a substantial amount of IFN- $\gamma$ compared with baseline and reached $60 \mathrm{pg} / \mathrm{ml}$ at 24 hours. (C) Titration assays demonstrate that HLA class I expression can be induced on LAN-1 and $\mathrm{BE}(2) \mathrm{N}$ cells with $10 \mathrm{pg} / \mathrm{ml} \mathrm{IFN}-\gamma$ and that expression increased in a dose-dependent manner.
(Figure 4A) to levels nearly equivalent to the level attained following the direct addition of IFN- $\gamma$ (Figure 3A). HLA-Bw4 expression was also specifically induced, albeit to a lower level than that induced in the other class I molecules.

We surmised that release of cytokines by tumor-activated PBMCs in the presence of $\mathrm{mAb}$ was responsible for the supernatant-mediated upregulation of HLA class I on the tumor target. Using ELISA, we determined the concentrations of IFN- $\gamma$ released in the different supernatants. PBMCs alone did not produce detectable IFN- $\gamma$, and the addition of GM-CSF resulted in the release of a minimal amount of IFN- $\gamma$ at 24 hours (Figure 4B). However, PBMCs activated by NB target cells in the presence of $3 \mathrm{~F} 8$ released a substantial amount of IFN- $\gamma$, peaking at 12 hours, and the addition of GMCSF to $3 F 8$ further enhanced IFN- $\gamma$ production, reaching $60 \mathrm{pg} / \mathrm{ml}$ by 24 hours (Figure 4B). Using titration assays, we determined that HLA expression on LAN-1 and BE(2)N cells could be induced with as little as $10 \mathrm{pg} / \mathrm{ml}$ IFN- $\gamma$ (Figure 4C). We therefore conclude that concentrations of IFN- $\gamma$ released by tumor-activated PBMCs in the presence of 3F8 and GM-CSF are adequate to induce HLA expression on the tumor.

\section{Discussion}

When treated with anti-GD2 mAb, patients with NB who lack one or more HLA ligand for their inhibitory KIRs respond better to treatment, have lower rates of relapse, and survive longer compared with patients who possess all HLA KIR ligands. These results are independent of stem cell transplantation and suggest that the missing ligand effect is a result of either the $3 \mathrm{~F} 8 \mathrm{mAb}$ therapy and/or the interaction between NK cells and the NB target. The response and survival advantages associated with missing KIR ligand genotypes, denoting individuals who lack HLA ligands for his/her autologous inhibitory KIR, suggested to us that the 


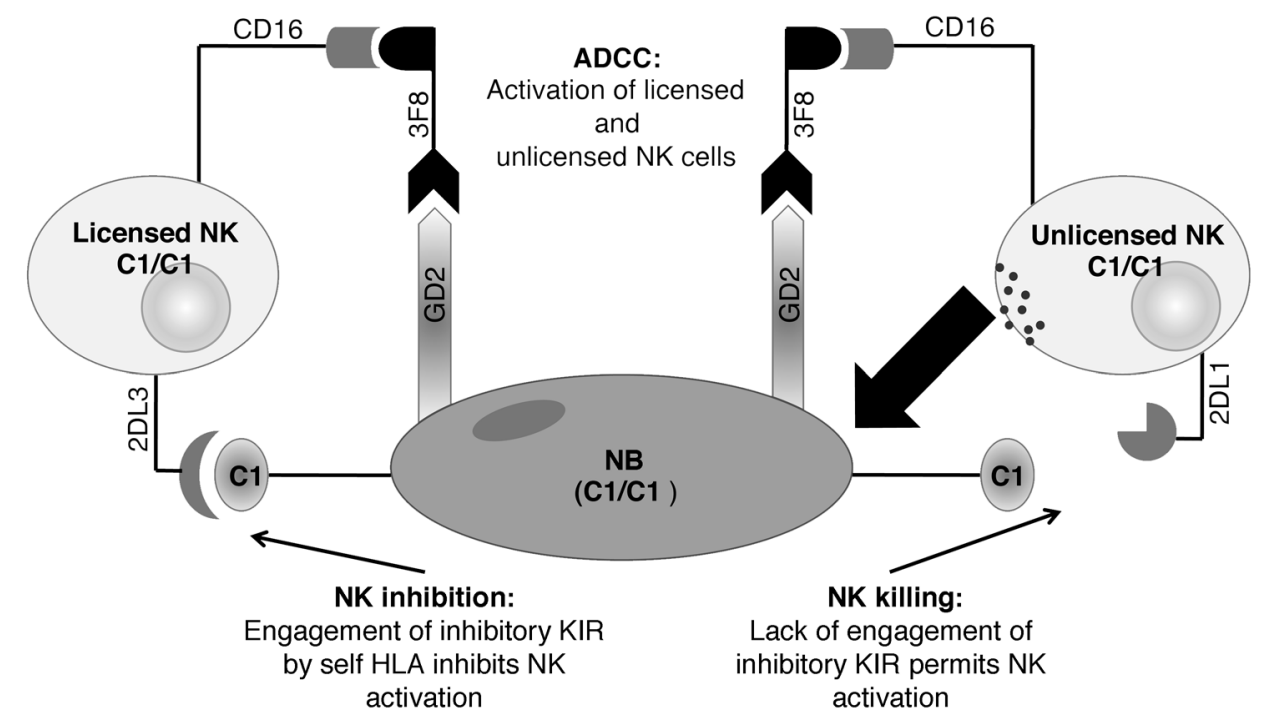

Figure 5

Unlicensed NK cells dominate NB killing in the presence of 3F8. 3F8 activates both licensed and unlicensed NK cells through CD16. Engagement of inhibitory KIR with its cognate HLA class I ligand on NB cells inhibits licensed NK cell-mediated killing, leaving activation of unlicensed NK cells expressing KIR for non-self or missing ligands intact.

unlicensed NK cells expressing KIRs for non-self-HLA ligands in these individuals may be more effective in tumor eradication than the licensed NK cells expressing KIRs for self HLA. We considered 2 possible mechanisms underlying these clinical findings: activation among the NS-KIR-specific NK population by 3F8 and/ or selective inhibition of the S-KIR-specific population. For NB treated with $3 \mathrm{~F} 8$, both mechanisms appear to be important.

In vitro, licensed NK cells expressing S-KIRs and unlicensed NK cells expressing NS-KIRs are both activated by NB targets in the presence of 3F8, but only the licensed cells are inhibited by cytokine-induced expression of self-HLA molecules on tumor targets. While NKG2A expression contributes to the ADCC response, NKG2A-bearing NK cells are also silenced by HLA-E expression on tumor cells $(27,35)$. Taken together, the data suggest that upon encountering target cells with HLA class I expression, unlicensed NK cells bearing receptors for non-self HLA are the primary mediators of ADCC. Several studies examining tumor samples from patients with NB and mouse models indicate that upregulation of HLA class I occurs in vivo. All patients in this study received chemotherapy, GM-CSF, and anti-GD2 mAb therapy, and most patients received isotretinoin. It has been reported that tumor specimens from patients with NB treated with chemotherapy upregulate class I expression as a result of partial differentiation (29); retinoid derivatives induce cellular differentiation and HLA class I expression on NB cells (30); and, in NB-bearing mice treated with anti-GD2 $\mathrm{mAb}$, recurrent tumors exhibit greater than 5-fold higher MHC class I expression (31). We could not demonstrate in vitro that agents used to treat NB could directly upregulate HLA class I expression on certain NB cell lines, suggesting that in vivo conditions may have contributed to previous findings or that the cell lines we used may not respond with HLA class I upregulation to these agents (30). We show clearly, however, that PBMCs responding to $\mathrm{NB}$ in the presence of $\mathrm{mAb}$ produce cytokines, in particular IFN- $\gamma$, at concentrations sufficient to induce HLA class I expression on NB cells. Other cytokines, such as tumor necrosis factor, can further enhance HLA class I expression, including HLABw4 (data not shown), and it is possible that these cytokines are also released in ADCC-dependent lymphocyte activation. These in vitro findings offer insight to in vivo conditions, in which recruitment of NK cells to the tumor microenvironment may result in effector/target interactions, leading to significant local cytokine concentrations. Taken together, the data indicate that it is highly likely that NK activation in vivo by $3 \mathrm{~F} 8$-dependent ADCC leads to cytokine release, inducing HLA class I expression on the NB target, inhibiting licensed NK cells, and facilitating the striking missing KIR ligand effect (Figure 5).

The in vitro studies presented in this report represented activity among NK cells expressing a single inhibitory KIR. Examination of the NK repertoire among 20 individuals revealed that the singlepositive unlicensed and licensed NK populations, while varying in size from individual to individual, can each represent a significant proportion of the total NK repertoire. The repertoire also includes smaller populations of NK cells that express two or more inhibitory receptors, often resulting in higher levels of responsiveness $(19,36)$. However, interaction between just one receptor and its HLA class I ligand is sufficient to inhibit these highly responsive NK cells (19). Thus, licensed NK cells expressing S-KIRs, whether expressing one or more than one inhibitory KIR, will be inactivated by the presence of self-HLA class I on the tumor target.

These findings are highly relevant to patients with other malignancies treated with $\mathrm{mAbs}$, such as rituximab, whose mechanisms of action involve NK-mediated ADCC (37). Higher HLA class I expression on CD20-positive lymphoma cells is associated with attenuation of rituximab-induced NK-mediated ADCC (38). However, correlation of KIR/HLA genotyping among patients receiving rituximab and other $\mathrm{mAb}$ therapies has not been systematically explored for the missing ligand effect.

Our data support the incorporation of KIR and HLA genotypes as a prognostic marker in patients with high-risk NB receiving antibody immunotherapy. Indeed, KIR/HLA genotypes are as 
strong a predictor of response and survival, if not better than several conventional NB biomarkers (25). An analogous study including only patients with $\mathrm{NB}$, particularly those who do not receive antibody treatment, should be performed to confirm whether $\mathrm{mAb}$ is necessary for this effect.

Since licensed NK cells expressing S-KIRs have higher ADCC capacity in general, rescuing licensed NK activity from class I inhibition is desirable and could increase response in all patients, including those with all KIR ligands present. Use of newly developed clinical antibodies directed to the inhibitory KIRs would prevent their engagement with HLA cognate ligands and potentially restore licensed NK function (39). In addition, the use of exogenous NK cells in the treatment of patients with NB may be potentially useful if the patient lacks class I ligands for the donor inhibitory KIRs. Augmentation of innate immunity through adoptive transfer of allogeneic NK cells or the use of agents that increase endogenous NK cell number and activity, such as IL-2, lenalidomide, and anti-CD137 antibody, may all improve NB control, particularly in the presence of 3F8 (40-42).

Unlicensed NK cells expressing NS-KIRs have a clinically significant impact in the control of acute myelogenous leukemia in allogeneic hematopoietic stem cell transplantation (23, 43, 44), and it is clear from this study that their impact extends to other tumor targets. While transplantation and $\mathrm{mAb}$ therapy are hardly normal physiologic conditions, they both take advantage of the important pool of unlicensed NK cells, previously thought to be hyporesponsive and therefore potentially less clinically relevant. In mice, unlicensed NK cells play a dominant role in clearing murine cytomegalovirus $(45,46)$, in which the proinflammatory cytokine environment of viral infection leads to higher function among unlicensed cells. These cells can be recalled upon viral rechallenge to eradicate infected cells more efficiently than licensed NK cells, whose cytotoxic and proliferative function is diminished in the presence of MHC class I expression $(45,46)$. It is possible that activity among unlicensed NK cells in patients with NB may also be augmented by the proinflammatory state associated with tumor burden. The existence of unlicensed NK cells that have been presumed to be hyporesponsive in an otherwise parsimoniously constructed human immune system has been a paradox. These findings contribute to our understanding of the critical role of the unlicensed NK population in tumor control.

\section{Methods}

Patients. This is a retrospective analysis of 242 patients with high-risk NB treated at Memorial Sloan-Kettering Cancer Center between 1994 and 2007 with anti-GD2 mAb 3F8. All but 1 patient received GM-CSF, 76\% of patients received oral isotretinoin, and 1 patient received oral $\beta$-glucan (http://clinicaltrial.gov/; study nos. NCT00072358, NCT00002560, and NCT00037011).

KIR and HLA genotyping. Genomic DNA, extracted from PBMCs or marrow mononuclear cells after remission using the QIAamp DNA Blood Mini Kit (Qiagen), was KIR genotyped using previously published methods (47). Patient HLA alleles were identified by a combination of HLA serology, sequence-based amplification (PCR sequence-specific primer), and oligonucleotide probing of genomic DNA (PCR-specific oligonucleotide probe). HLA genotyping for healthy donors was performed at HistoGenetics.

Target cells, effector cells, and culture conditions. The human erythroleukemia cell line K562 (ATCC) and human NB cell lines IMR-32 (ATCC) and LAN-1 (provided by Robert Seeger, Children's Hospital Los Angeles, Los Angeles, California, USA) as well as the human NB cell line NB-1691 (13) (provided by Peter Houghton, Nationwide Children's Research Institute Center for Childhood Cancer, Columbus, Ohio, USA) and 3 cell lines, BE(1)N, BE(2) $\mathrm{N}$, and SKNLP, derived from patients with NB treated at Memorial SloanKettering Cancer Center were cultured in RPMI-1640 medium supplemented with $10 \%$ heat-inactivated fetal bovine serum, $100 \mathrm{U} / \mathrm{ml}$ penicillin, $100 \mu \mathrm{g} / \mathrm{ml}$ streptomycin, $1 \%$ sodium pyruvate, and $1 \% 2$-mercaptoethanol and incubated at $37^{\circ} \mathrm{C}$ with $5 \% \mathrm{CO}_{2}$. To upregulate HLA class I expression, recombinant human IFN- $\gamma$ (PeproTech) was added at 1,000 units $/ \mathrm{ml}$ for 72 hours (33). For supernatant experiments, PBMCs $\left(1 \times 10^{5}\right.$ cells per well $)$ were placed in culture alone or with NB cell lines at a ratio of $1: 1$, with or without $3 \mathrm{~F} 8$ and GM-CSF at $2 \mathrm{ng} / \mathrm{ml}$ (34) for 24 hours, in 96-well U-bottom plates with $200 \mu \mathrm{l}$ of medium, described above, per well. The supernatant was collected, centrifuged, filtered through a $22-\mu \mathrm{m}$ filter (Millipore), and then used as culture media for the same NB cell lines for 72 hours prior to assaying HLA expression. To test induction of HLA class I expression by therapeutic agents, NB cell lines were cultured in complete medium in the presence or absence of vincristine $(50 \mathrm{ng} / \mathrm{ml}$ and $200 \mathrm{ng} / \mathrm{ml})$, thiotepa $(20 \mu \mathrm{g} / \mathrm{ml}$ and $200 \mathrm{ng} / \mathrm{ml})$, and doxorubicin $(1 \mu \mathrm{g} / \mathrm{ml})$ for 48 hours; isotretinoin $(5 \mu \mathrm{M})$ for 72 hours; or GM-CSF $(2 \mathrm{ng} / \mathrm{ml})$ for 72 hours prior to assaying HLA expression. Both PBMCs and purified NK cells from healthy individuals were used for analysis of NK function. PBMCs were isolated by Ficoll-density gradient centrifugation. NK cells were isolated following $\mathrm{CD}^{+}$depletion and $\mathrm{CD}^{+} 6^{+}$enrichment using the CliniMACS system (Miltenyi). Effector cells were cultured in medium, described above, supplemented with human IL-2 (Proleukin, Prometheus) at 1,000 U/ml and incubated at $37^{\circ} \mathrm{C}$ with $5 \% \mathrm{CO}_{2}$ for 12 to 16 hours prior to assaying function.

NK activation and flow cytometric analysis. CD107 mobilization and intracellular IFN- $\gamma$ production were used as indicators of effector cell activation (48-50). PBMCs $\left(5 \times 10^{5}\right.$ cells per well $)$ or NK cells $\left(2 \times 10^{5}\right.$ cells per well) were incubated with $\mathrm{NB}$ target cells at ratios of $1: 3$ and $1: 1$, respectively, with or without $0.8 \mu \mathrm{g} / \mathrm{ml} 3 \mathrm{~F} 8$ for 4 hours in 96-well U-bottom plates with $200 \mu \mathrm{l}$ medium, described above, per well. The 3F8 concentration was selected after titration assays demonstrated no difference in in vitro NK activation at higher concentrations (Supplemental Figure 4) and is less than the serum concentration achieved in patients treated with 3F8 (1-4 $\mu \mathrm{g} / \mathrm{ml})(51)$. For HLA class I blocking, NB cells were preincubated for 30 minutes at $37^{\circ} \mathrm{C}$ with an antibody cocktail composed of DX17 (BD Biosciences), and 6A4 and A6.136 (both provided by Alessandro Moretta, Università di Genova, Genoa, Italy) (52) prior to the addition of PBMCs or purified NK cells and 3F8. Isotype controls were used for each sample. PerCPCy5.5-conjugated anti-CD107a or Pacific Blue-conjugated anti-CD107a (H4A3 clone, Biolegend) was added to each well prior to incubation. Cells were stained with anti-CD3-APC-Cy7 (clone SK7, BD Biosciences), anti-CD56-PE-Cy7 (clone B159, BD Biosciences), or antiCD56-ECD (clone N901, Beckman Coulter); anti-KIR2DL1/2DS1-APC (clone HP-MA4, eBioscience), anti-KIR2DL2/2DL3/2DS2-FITC (clone CH-L, BD Biosciences), anti-KIR3DL1-PE (clone DX9, BD Biosciences), or anti-KIR3DL1-Alexa Fluor 700 (clone DX9, Biolegend); anti-NKG2A-PE (clone Z199, Beckman Coulter); or Pacific Blue-conjugated anti-NKG2A (clone Z199, Beckman Coulter). For IFN- $\gamma$ evaluation, brefeldin-A (10 $\mu \mathrm{g} / \mathrm{ml}$, Sigma-Aldrich) and GolgiStop ( $6 \mu \mathrm{g} / \mathrm{ml}$, BD Biosciences) were added to the mixture 1 hour following incubation, and FIX \& PERM (Invitrogen) was used for staining. LIVE/DEAD Aqua Fixable Dead Cell Stains (Invitrogen) were used to exclude dead cells from analysis. Cells were analyzed using multicolor flow cytometry on a LSRII instrument with FACS Diva software (BD Biosciences). Results were interpreted using FlowJo software (Tree Star). For multicolor compensation and gating, unstained, single-color, and FMO controls were used. NK cells exclusively expressing a single inhibitory KIR were evaluated as previously described $(19,22)$. HLA class I and GD2 expression on NB cells was evaluated with 
anti-HLA-ABC-FITC (clone G46-2.6, BD Biosciences), anti-HLA-Bw4-FITC (clone FH007, One Lambda), anti-HLA-E-PE (clone 3D12, Biolegend), and anti-GD2-Alexa Fluor 488 (clone 2Q549, Abcam).

NK cytotoxicity. Cell-mediated cytotoxicity was analyzed by flow cytometry with the PKH26 membrane dye and TO-PRO-3 DNA label as previously described (19), with and without 3F8.

Cytokine assay. IFN- $\gamma$ production was measured by ELISA (R\&D Systems) according to the manufacturer's instructions and analyzed using an MRX plate reader (Dynex Technology) and Biolinx 2.22 software (Dynex Technology).

Statistics. OS was defined as the time from 3F8 therapy to the date of death or last follow-up. PFS was defined as the time from $3 \mathrm{~F} 8$ therapy to the date of relapse, death, or last follow up. Survival probabilities were estimated using the Kaplan-Meier method. The log-rank test was used to compare survival probabilities among patients missing any or possessing all KIR ligands. HRs of missing KIR ligand indicators were estimated by multivariable Cox regression, controlling for age, MYCN amplification, and bone disease. Fisher's exact test was used to determine whether the disease status distribution differed between the missing ligand and all ligands present patient groups. To investigate activation of licensed and unlicensed NK cells, CD107 mobilization and intracellular IFN- $\gamma$ were measured for each population following incubation with NB cell lines in the presence or absence of 3F8. Paired 2-tailed Student's $t$ test was used to compute the comparisons within the licensed or unlicensed populations, and the unpaired 2-tailed Student's $t$ test was used to compute comparisons between the unlicensed and licensed populations. For relative fold increase calculations, the unpaired Student's $t$ test was used to compare the relative activity of licensed and unlicensed NK cells. A test with a $P$ value of less than 0.05 was considered statistically significant.
Study approval. Informed consent for specimen collection was obtained from patients or legal guardians in accordance with institutional review board guidelines at Memorial Sloan-Kettering Cancer Center. These studies were approved by the institutional review board of Memorial SloanKettering Cancer Center.

\section{Acknowledgments}

We acknowledge the neuroblastoma team at Memorial SloanKettering Cancer Center and Marcel Van Den Brink for use of the LSRII flow cytometer. This study was supported in part by Alex's Lemonade Stand Foundation, the NIH (HL088134, CA083766, and CA106450), the CALGB Clinical Scholars Award, the Robert Steel Foundation, and Katie Find a Cure. We acknowledge the support Novartis Oncology, provided through the CALGB Foundation.

Received for publication January 9, 2012, and accepted in revised form June 28, 2012.

Address correspondence to: Katharine C. Hsu, Department of Medicine, Memorial Sloan-Kettering Cancer Center, 1275 York Ave., New York, New York 10065, USA. Phone: 646.888.2667; Fax: 646.422.0298; E-mail: hsuk@mskcc.org.

Nidale Tarek's present address is: Department of Pediatrics, The University of Texas MD Anderson Cancer Center, Houston, Texas, USA.

Jeffrey M. Venstrom's present address is: Department of Medicine, UCSF, San Francisco, California, USA.
1. Matthay KK, et al. Treatment of high-risk neuroblastoma with intensive chemotherapy, radiotherapy, autologous bone marrow transplantation, and 13-cis-retinoic acid. $N$ Engl J Med. 1999;341(16):1165-1173.

2. Berthold F, et al. Myeloablative megatherapy with autologous stem-cell rescue versus oral maintenance chemotherapy as consolidation treatment in patients with high-risk neuroblastoma: a randomised controlled trial. Lancet Oncol. 2005;6(9):649-658.

3. Cheung NK, et al. Anti-G(D2) antibody treatment of minimal residual stage 4 neuroblastoma diagnosed at more than 1 year of age. J Clin Oncol. 1998;16(9):3053-3060.

4. Modak S, Cheung NK. Neuroblastoma: Therapeutic strategies for a clinical enigma. Cancer Treat Rev. 2010;36(4):307-317.

5. Yu AL, et al. Anti-GD2 antibody with GM-CSF, interleukin-2, and isotretinoin for neuroblastoma. N Engl J Med. 2010;363(14):1324-1334.

6. Vivier E, Tomasello E, Baratin M, Walzer T, Ugolini S. Functions of natural killer cells. Nat Immunol. 2008;9(5):503-510.

7. Wolfl M, et al. Expression of MHC class I, MHC class II, and cancer germline antigens in neuroblastoma. Cancer Immunol Immunother. 2005;54(4):400-406.

8. Raffaghello L, et al. Mechanisms of immune evasion of human neuroblastoma. Cancer Lett. 2005;228(1-2):155-161.

9. Sivori S, et al. Involvement of natural cytotoxicity receptors in human natural killer cell-mediated lysis of neuroblastoma and glioblastoma cell lines. J Neuroimmunol. 2000;107(2):220-225.

10. Castriconi R, et al. Natural killer cell-mediated killing of freshly isolated neuroblastoma cells: critical role of DNAX accessory molecule-1-poliovirus receptor interaction. Cancer Res. 2004;64(24):9180-9184.

11. Ogbomo H, Hahn A, Geiler J, Michaelis M, Doerr
HW, Cinatl J. NK sensitivity of neuroblastoma cells determined by a highly sensitive coupled luminescent method. Biochem Biophys Res Commun. 2006;339(1):375-379.

12. Modak S, Kramer K, Gultekin SH, Guo HF, Cheung NK. Monoclonal antibody 8H9 targets a novel cell surface antigen expressed by a wide spectrum of human solid tumors. Cancer Res. 2001;61(10):4048-4054.

13. Xu H, Cheung IY, Guo HF, Cheung NK. MicroRNA miR-29 modulates expression of immunoinhibitory molecule B7-H3: potential implications for immune based therapy of human solid tumors. Cancer Res. 2009;69(15):6275-6281.

14. Castriconi R, et al. Identification of $4 \mathrm{Ig}-\mathrm{B} 7-\mathrm{H} 3$ as a neuroblastoma-associated molecule that exerts a protective role from an NK cell-mediated lysis. Proc Natl Acad Sci U S A. 2004;101(34):12640-12645.

15. Lanier LL. NK cell recognition. Annu Rev Immunol. 2005;23:225-274.

16. Moesta AK, Norman PJ, Yawata M, Yawata N, Gleimer M, Parham P. Synergistic polymorphism at two positions distal to the ligand-binding site makes KIR2DL2 a stronger receptor for HLA-C than KIR2DL3. J Immunol. 2008;180(6):3969-3979.

17. Kim S, et al. Licensing of natural killer cells by host major histocompatibility complex class I molecules. Nature. 2005;436(7051):709-713.

18. Anfossi N, et al. Human NK cell education by inhibitory receptors for MHC class I. Immunity. 2006;25(2):331-342.

19. Yu J, Heller G, Chewning J, Kim S, Yokoyama WM, Hsu KC. Hierarchy of the human natural killer cell response is determined by class and quantity of inhibitory receptors for self-HLA-B and HLA-C ligands. J Immunol. 2007;179(9):5977-5989.

20. Yokoyama W, Kim S. Licensing of natural killer cells by self-major histocompatibility complex class I. Immunol Rev. 2006;214:143-154.
21. Fernandez N, Treiner E, Vance R, Jamieson A, Lemieux S, Raulet D. A subset of natural killer cells achieves self-tolerance without expressing inhibitory receptors specific for self-MHC molecules. Blood. 2005;105(11):4416-4423.

22. Yu J, et al. Breaking tolerance to self, circulating natural killer cells expressing inhibitory KIR for non-self HLA exhibit effector function after T celldepleted allogeneic hematopoietic cell transplantation. Blood. 2009;113(16):3875-3884.

23. Hsu KC, et al. Improved outcome in HLA-identical sibling hematopoietic stem-cell transplantation for acute myelogenous leukemia predicted by KIR and HLA genotypes. Blood. 2005;105(12):4878-4884.

24. Parham P. MHC class I molecules and KIRs in human history, health and survival. Nat Rev Immunol. 2005;5(3):201-214.

25. Venstrom JM, et al. KIR and HLA genotypes are associated with disease progression and survival following autologous hematopoietic stem cell transplantation for high-risk neuroblastoma. Clin Cancer Res. 2009;15(23):7330-7334.

26. Delgado DC, et al. Genotypes of NK cell KIR receptors, their ligands, and Fcgamma receptors in the response of neuroblastoma patients to Hu14. Cancer Res. 2010;70(23):9554-9561.

27. Bezlat V, Descours B, Parizot C, Debre P, Vieillard $V$. NK cell terminal differentiation: correlated stepwise decrease of NKG2A and acquisition of KIRs. PLoS One. 2010;5(8):e11966.

28. Cooper M, Fehniger T, Caligiuri M. The biology of human natural killer-cell subsets. Trends Immunol. 2001;22(11):633-640

29. Favrot MC, et al. Expression of leucocyte adhesion molecules on 66 clinical neuroblastoma specimens. Int J Cancer. 1991;48(4):502-510.

30. Vertuani S, De Geer A, Levitsky V, Kogner P, Kiessling R, Levitskaya J. Retinoids act as multistep modulators of the major histocompatibility 
class I presentation pathway and sensitize neuroblastomas to cytotoxic lymphocytes. Cancer Res. 2003;63(22):8006-8013.

31. Neal ZC, et al. NXS2 murine neuroblastomas express increased levels of MHC class I antigens upon recurrence following NK-dependent immunotherapy. Cancer Immunol Immunother. 2004;53(1):41-52.

32. Carlson LM, Pahlman S, De Geer A, Kogner P, Levitskaya J. Differentiation induced by physiological and pharmacological stimuli leads to increased antigenicity of human neuroblastoma cells. Cell Res. 2008;18(3):398-411.

33. Lampson LA, Fisher CA. Weak HLA and beta 2-microglobulin expression of neuronal cell lines can be modulated by interferon. Proc Natl Acad Sci US A. 1984;81(20):6476-6480.

34. Kushner B, Cheung N. GM-CSF enhances 3F8 monoclonal antibody-dependent cellular cytotoxicity against human melanoma and neuroblastoma. Blood. 1989;73(7):1936-1941.

35. Borrego F, Ulbrecht M, Weiss EH, Coligan JE, Brooks AG. Recognition of human histocompatibility leukocyte antigen (HLA)-E complexed with HLA class I signal sequence-derived peptides by CD94/NKG2 confers protection from natural killer cell-mediated lysis. J Exp Med. 1998;187(5):813-818.

36. Joncker N, Fernandez N, Treiner E, Vivier E, Raulet D. NK cell responsiveness is tuned commensurate with the number of inhibitory receptors for self-MHC class I: the rheostat model. J Immunol. 2009;182(8):4572-4580.

37. Weiner G. Rituximab: mechanism of action. Semin
Hematol. 2010;47(2):115-123.

38. Borgerding A, et al. B-lymphoma cells escape rituximab-triggered elimination by NK cells through increased HLA class I expression. Exp Hematol. 2010;38(3):213-221.

39. Romagne F, et al. Preclinical characterization of 1-7F9, a novel human anti-KIR receptor therapeutic antibody that augments natural killer-mediated killing of tumor cells. Blood. 2009;114(13):2667-2677.

40. Ladenstein R, et al. Dose finding study for the use of subcutaneous recombinant interleukin-2 to augment natural killer cell numbers in an outpatient setting for stage 4 neuroblastoma after megatherapy and autologous stem-cell reinfusion. J Clin Oncol. 2011;29(4):441-448.

41. Wu L, Parton A, Lu L, Adams M, Schafer P, Bartlett JB. Lenalidomide enhances antibody-dependent cellular cytotoxicity of solid tumor cells in vitro: influence of host immune and tumor markers. Cancer Immunol Immunother. 2011;60(1):61-73.

42. Kohrt HE, et al. CD137 stimulation enhances the antilymphoma activity of anti-CD20 antibodies. Blood. 2011;117(8):2423-2432.

43. Beelen DW, et al. Genotypic inhibitory killer immunoglobulin-like receptor ligand incompatibility enhances the long-term antileukemic effect of unmodified allogeneic hematopoietic stem cell transplantation in patients with myeloid leukemias. Blood. 2005;105(6):2594-2600.

44. Miller JS, et al. Missing KIR ligands are associated with less relapse and increased graft-versus-host disease (GVHD) following unrelated donor allogeneic HCT. Blood. 2007;109(11):5058-5061.
45. Sun JC, Lanier LL. Cutting edge: viral infection breaks NK cell tolerance to "missing self". J Immunol. 2008;181(11):7453-7457.

46. Orr MT, Murphy WJ, Lanier LL. 'Unlicensed' natural killer cells dominate the response to cytomegalovirus infection. Nat Immunol. 2010;11(4):321-327.

47. Hsu KC, Liu XR, Selvakumar A, Mickelson E, O'Reilly RJ, Dupont B. Killer Ig-like receptor haplotype analysis by gene content: evidence for genomic diversity with a minimum of six basic framework haplotypes, each with multiple subsets. J Immunol. 2002;169(9):5118-5129.

48. Bryceson YT, March ME, Barber DF, Ljunggren HG, Long EO. Cytolytic granule polarization and degranulation controlled by different receptors in resting NK cells. J Exp Med. 2005;202(7):1001-1012.

49. Rubio V, et al. Ex vivo identification, isolation and analysis of tumor-cytolytic T cells. Nat Med. 2003;9(11):1377-1382.

50. Wolint P, Betts MR, Koup RA, Oxenius A. Immediate cytotoxicity but not degranulation distinguishes effector and memory subsets of CD8+ T cells. J Exp Med. 2004;199(7):925-936.

51. Cheung NK, et al. Ganglioside GD2 specific monoclonal antibody 3F8: a phase I study in patients with neuroblastoma and malignant melanoma. J Clin Oncol. 1987;5(9):1430-1440.

52. Ciccone E, et al. General role of HLA class I molecules in the protection of target cells from lysis by natural killer cells: evidence that the free heavy chains of class I molecules are not sufficient to mediate the protective effect. Int Immunol. 1995;7(3):393-400. 